\title{
Carbon dioxide clathrate hydrate FTIR spectrum
}

\section{Near infrared combination modes for astrophysical remote detection}

\author{
E. Dartois ${ }^{1, \star}$ and B. Schmitt ${ }^{2}$ \\ 1 Institut d'Astrophysique Spatiale, UMR-8617, Université Paris-Sud, bâtiment 121, 91405 Orsay, France \\ e-mail: emmanuel.dartois@ias.u-psud.fr \\ ${ }^{2}$ Laboratoire de Planétologie de Grenoble, Bâtiment D de Physique, Domaine Universitaire, BP 53, 38041 Grenoble Cedex 9 , France
}

Received 9 February 2009 / Accepted 11 June 2009

\begin{abstract}
Context. Clathrate hydrates could provide a sink for highly volatile molecules, thus modifying the release and chemical cycling time scales for gases in icy bodies in the solar system (planets, satellites, comets), as well as for interstellar ice mantles.

Aims. By providing an infrared spectroscopic identification for the carbon dioxide clathrate hydrate, $\mathrm{CO}_{2}$ being an important constituent of ices in interstellar (ISM) and planetary media, we examine its astrophysical presence or absence.

Methods. A carbon dioxide clathrate crystal is produced in an infrared transmitting moderate pressure closed cell. Using FTIR spectroscopy, the stretching modes $\left(v_{3}^{12} \mathrm{CO}_{2},{ }^{13} \mathrm{CO}_{2},{ }^{18} \mathrm{OCO}\right)$ and accidental resonances combinations $\left({ }^{12} \mathrm{CO}_{2},{ }^{13} \mathrm{CO}_{2},{ }^{18} \mathrm{OCO} v_{1}+v_{3} \mathrm{Fermi}\right.$ resonance dyad and $2 v_{1}+v_{3}$ Fermi resonance triad) falling in the 5100-2200 $\mathrm{cm}^{-1}(1.96-4.43 \mu \mathrm{m})$ range, and their temperature behaviour from $150 \mathrm{~K}$ down to $5.6 \mathrm{~K}$ are investigated.

Results. Combination modes clearly show the two distinct cages expected for type I carbon dioxide clathrate hydrate, and we identify them. The forbidden antisymmetric stretching-mode overtone $\left(2 v_{3}\right)$, activated in the carbon dioxide simple hydrate, is absent in the clathrate hydrate. Combining these distinct spectroscopic profiles will provide a constraint to determine the importance of carbon dioxide clathrate hydrates observationally.

Conclusions. We spectroscopically identify the carbon dioxide clathrate hydrate. A direct detection via (near-)infrared probes or telescopic observations is needed to understand whether clathrate formation is ubiquitous, given the widespread occurrence of carbon dioxide and water ice in astrophysics, or whether it is present only very locally in a few objects.
\end{abstract}

Key words. line: profiles - comets: general - solar system: general - ISM: dust, extinction - ISM: lines and bands line: identification

\section{Introduction}

Clathrate hydrates are icelike compounds with an H-bonded water ice network hosting guest molecules. Carbon dioxide clathrate hydrate has been investigated for its ability to be epitaxially grown in so-called type I or type II structures (Fleyfel \& Devlin 1991). These two structures differ in the size and number of water cages, hence in the potential interaction between the encaged $\mathrm{CO}_{2}$ and the water cage. As a consequence, the carbon dioxide main antisymmetric $\left(v_{3}\right)$ and bending $\left(v_{2}\right)$ vibration modes provide a unique pattern for identifying which structure is formed.

This cage-compound phase deserves to be investigated in astrophysics because carbon dioxide is the dominant constituent of the Martian atmosphere and seasonaly condenses in large amounts at high latitudes. Water is a minor constituent of the atmosphere and water ice is also detected in small amounts both as clouds and in the seasonal $\mathrm{CO}_{2}$-rich condensates. However both polar caps are mostly composed of water ice, and very large ice expanses are also detected as permafrost just below a dust layer of some tens centimetres (e.g. Boynton et al. 2002; Barr \& Milkovich 2008; Tamparri et al. 2008; Demidov et al. 2008; Mouginot et al. 2009). Since the first study by

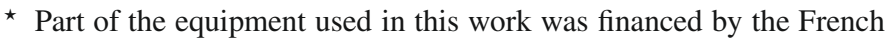
INSU-CNRS program "Physique et Chimie du Milieu Interstellaire" (PCMI). This work benefitted from the NIST WebBook, ADS abstract services, Biblioplanets, and ISI Web of knowledge databases
}

Miller \& Smythe (1970), these observations have raised interest in examining the $\mathrm{CO}_{2}-\mathrm{H}_{2} \mathrm{O}$ system thermodynamics in detail (e.g. Longhi 2005). In particular, under current Martian conditions, pure carbon dioxide solid and clathrate hydrate phases can coexist at the surface at high latitudes and possibly also with liquid $\mathrm{CO}_{2}$ in specific conditions at some depth (e.g. Longhi 2006). The kinetics of carbon dioxide clathrate hydrate formation (Schmitt 1986; Schmitt et al. 2003; Circone et al. 2003; Genov 2003) and the thermodynamic stability curves (e.g. Lunine \& Stevenson 1985; Fray et al. 2009) are, in addition to the spectroscopic characterisation we focus on in this article, key aspects needed to understand its formation conditions.

Carbon dioxide is also a significant constituent of comets. It has been directly observed in the gas phase in four comets (Colangeli et al. 1999; Combes et al. 1988; Crovisier et al. 1997; Feaga et al. 2007) and is also infered from the CO Cameron excited band resulting from the photodestruction of $\mathrm{CO}_{2}$ molecules (Weaver et al. 1994; Feldman et al. 1997). It has also been detected at the surface of several satellites such as the Jovian Europa, Ganymede, and Callisto, Saturn's Iapetus, Phoebe, and Hyperion, the Uranian Ariel, Umbriel, and Titania, and Neptune's Triton (e.g. Brown et al. 2006; Buratti et al. 2005; Clark et al. 2005; Hibbitts et al. 2000; Hibbitts et al. 2003; Quirico et al. 1999). Farther away in dense ISM clouds, carbon dioxide represents in interstellar ice mantles, remarkably, almost always about one fifth of the water ice mantle content (e.g. de Graauw et al. 1996; Pontoppidan et al. 2008). Given the large 
panel of astrophysical objects where $\mathrm{CO}_{2}$ is a major species, the spectroscopic identification of possible $\mathrm{CO}_{2}$ clathrate hydrate on these objects is interesting beause of its strong implication in the formation conditions of these compounds, their inventory, and their equilibrium and exchange with the gas phase. In this article we study the spectroscopic signature in the near infrared of the type I carbon dioxide clathrate hydrate between $5.6 \mathrm{~K}$ and $150 \mathrm{~K}$.

\section{Experiments}

An evacuable closed cell was used, with two $\mathrm{ZnSe}$ windows for infrared transmission analysis, as described in our previous clathrate hydrate experiments (Dartois \& Deboffle 2008). The cell was thermally coupled to a liquid He-transfer cold finger, placed in a high-vacuum, evacuated cryostat $\left(P<10^{-7} \mathrm{mbar}\right)$. A stainless steel injection tube for gases entrance or evacuation was sealed at the bottom of the cell and ended with a four-port injector inside.

The formation of the carbon dioxide clathrate hydrate follows a procedure previously described for methane clathrate hydrate (Dartois \& Deboffle 2008) in which the ice film is formed first and pressurised with carbon dioxide gas during approximately two days in a region of the $(P, T)$ diagram above the equilibrium curve for carbon dioxide clathrate hydrate (20 bars, $255 \mathrm{~K})$. Then the temperature is decreased while progressively evacuating the excess $\mathrm{CO}_{2}$, taking care to maintain the system just below the vaporisation or sublimation curves of the $\mathrm{CO}_{2}$ phase diagram to avoid both the decomposition of the clathrate and condensation of some $\mathrm{CO}_{2}$ ice. FTIR spectra were recorded with a Bruker IFS $66 \mathrm{v}$ at a resolution of $0.5 \mathrm{~cm}^{-1}$, with a tungsten filament source, $\mathrm{KBr}$ beamsplitter and an $\mathrm{HgCdTe}$ detector cooled with liquid $\mathrm{N}_{2}$. Several experiments were conducted in order to produce carbon dioxide clathrate samples with different thicknesses to maximise the absorptions in each region of the spectrum. Among these experiments, we selected the best experimental set of spectra recorded between $150 \mathrm{~K}$ to $5.6 \mathrm{~K}$.

\section{Results}

\subsection{Carbon dioxide clathrate hydrate identification}

The formation of carbon dioxide clathrate hydrate is checked and identified via the $v_{3}$ vibration mode. Fleyfel \& Devlin (1991) have grown epitaxially $\mathrm{CO}_{2}$ clathrate hydrates on the previously grown, structure I or II clathrate hydrates of other molecules and recorded their spectra in the main infrared transitions. The absorption band positions observed in our experiments, shown in Fig. 1, at 2280 and $2271 \mathrm{~cm}^{-1}$, closely follow their type ${ }^{13} \mathrm{CO}_{2}$ clathrate FTIR spectrum for molecules trapped in the small and large cages, respectively. The main isotopomer transitions at approximately 2335 and $2347 \mathrm{~cm}^{-1}$ are saturated in our spectra. The very weak shoulder seen around $2320 \mathrm{~cm}^{-1}$ at $13 \mathrm{~K}$ in Fig. 2 of Fleyfel \& Devlin develops in our low-temperature spectra into an individual band that we attribute to ${ }^{18} \mathrm{O}$-substituted carbon dioxide, based on shifts measured in the gas phase (Rothman 1986). The second band, expected at a slightly higher wavenumber for $\mathrm{C}^{18} \mathrm{O}^{16} \mathrm{O}$ trapped in the second cage type, can be hardly seen in our spectra because of the strong overlap with the saturated main $\mathrm{CO}_{2}$ bands (Fig. 1). A large band better seen above $2355 \mathrm{~cm}^{-1}$ is present and corresponds to the $3 v_{L}$ harmonic of the water ice libration mode.

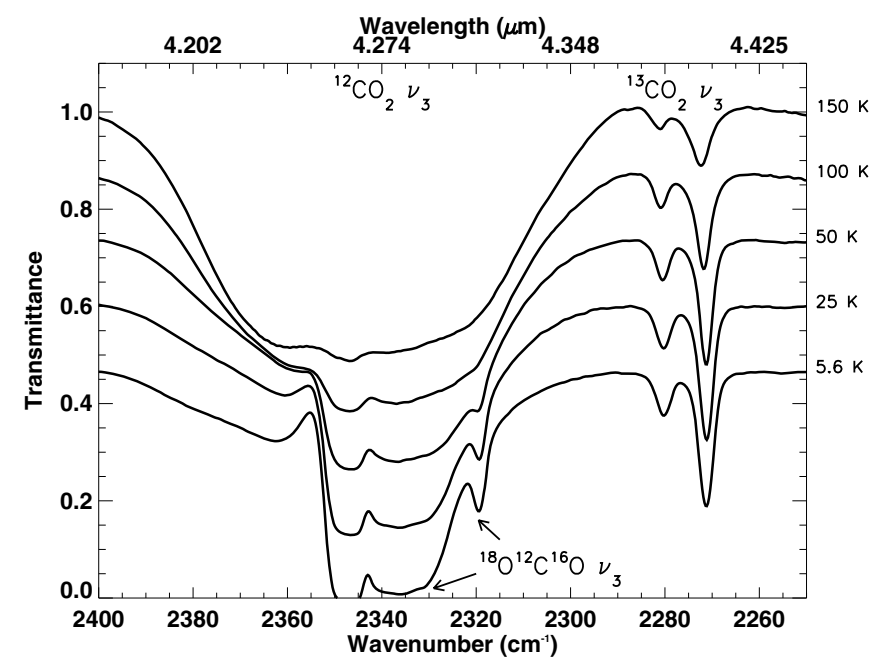

Fig. 1. Temperature-dependent spectrum in the ${ }^{12} \mathrm{CO}_{2}$ and ${ }^{13} \mathrm{CO}_{2} v_{3}$ antisymmetric, stretching-mode, vibrational region for the clathrate hydrate formed. Spectra are shifted vertically for clarity.

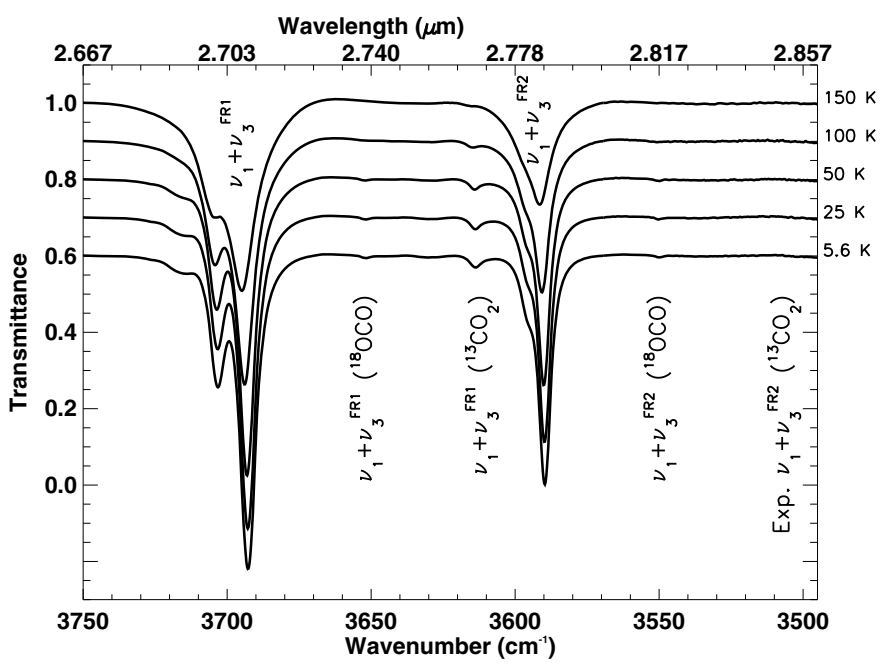

Fig. 2. Temperature-dependent spectra of carbon dioxide clathrate hydrate in the region of the $v_{1}+v_{3}$ Fermi resonance combination modes. Spectra are shifted vertically for clarity.

\subsection{Near-infrared spectra of carbon dioxide clathrate hydrate}

The $\mathrm{CO}_{2}$ clathrate hydrate, near-infrared spectra covering the strongest combination transitions in the $5100-3550 \mathrm{~cm}^{-1}$ range are presented in Figs. 2 and 3, i.e. covering the $v_{1}+v_{3}$ and $2 v_{1}+v_{3}$ Fermi resonances. The weak ${ }^{13} \mathrm{CO}_{2}$ and ${ }^{18} \mathrm{OCO}$ transitions in the first Fermi resonance range were tentatively assigned, based on natural isotopomer abundances and on the shift of the bands for the gas phase species (Rothman 1986). We adopted the same notation, assigning the Fermi resonance ranking (FRi where $i=1,2,3 \ldots)$ index starting from the highest level of the resonance. The $v_{1}+v_{3} \mathrm{FR} 2{ }^{13} \mathrm{CO}_{2}$ transitions are not detected in the present experiments, and only an expected position is given. It may partly stem from the onset of the strong absorption wing from the water ice network, removed by the adopted continuum, but the intensity ratio of the Fermi dyad for this isotope nevertheless seems very different from the main isotope equivalent transitions. An additional absorption was observed at about $3714 \mathrm{~cm}^{-1}$. Among the questions to address about the origin of this band, this may imply an interaction of the $\mathrm{CO}_{2} v_{3}$ mode with 


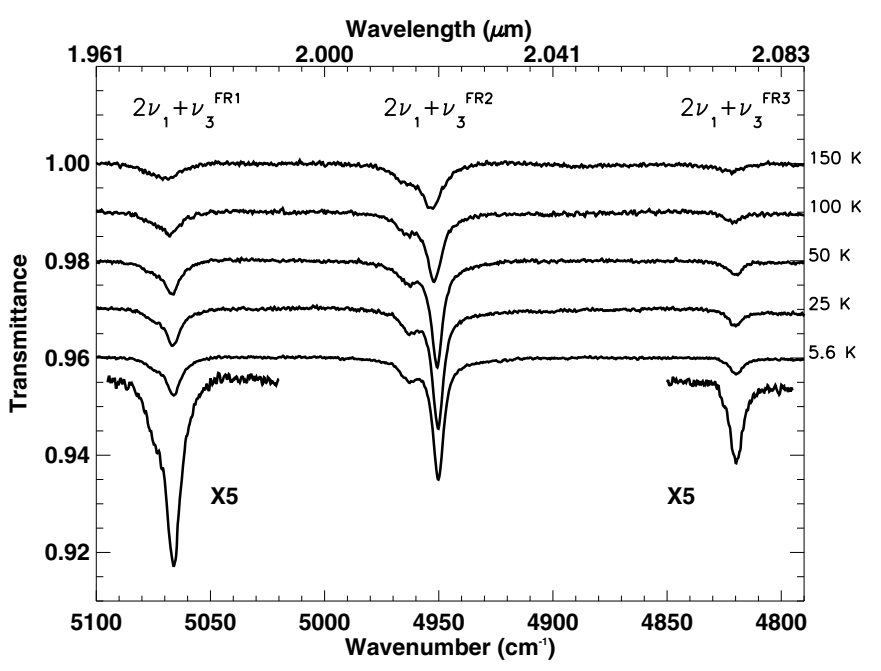

Fig. 3. Temperature-dependent spectra of carbon dioxide clathrate hydrate in the $2 v_{1}+v_{3}$ Fermi resonance combination modes absorption range. Spectra are shifted vertically for clarity.

the water ice libration harmonic as seen at shorter wavenumbers. A transition associated with, e.g., $\mathrm{CO}_{2}$ double occupancy for the same cages is very unlikely, given the large size of this molecule with respect to type I clathrate cages. From $150 \mathrm{~K}$ to $5.6 \mathrm{~K}$, we observe the spectral clarification associated to the sharpening of the individual enclathrated $\mathrm{CO}_{2}$ bands, accompanied by an intensity increase and a shift towards slightly lower frequencies. For the ${ }^{13} \mathrm{CO}_{2} v_{3}$ mode, the integrated absorbance increases by a factor of about 3 between $150 \mathrm{~K}$ and $5.6 \mathrm{~K}$, betraying the polarisability induced by the cages on the asymmetric stretching mode, a characteristic encountered in various degrees in clathrate hydrates.

We summarise the observed positions at $5.6 \mathrm{~K}$ for the carbon dioxide, type I clathrate hydrate in Table 1, and compare them to three other phases: $\mathrm{CO}_{2}$ in the gas phase, in its pure solid phase, and $\mathrm{CO}_{2}$ as a simple hydrate when mixed at low temperature in amorphous $\mathrm{H}_{2} \mathrm{O}$ ice. In addition to the band splitting induced by the trapping of $\mathrm{CO}_{2}$ in the two different cages of the type I clathrate hydrate structure, a fundamental difference between the clathrate hydrate and the simple hydrate spectra is the absence of activation of the $2 v_{3}$ mode in clathrate hydrate, while a strong $4678 \mathrm{~cm}^{-1}$ band is observed when the hydrate is formed (e.g. Fig. 3 of Bernstein et al. 2005).

\subsection{Phase diagrams}

Pure $\mathrm{CO}_{2}$ ice forms a peculiar thermodynamical pair with its clathrate hydrate form. Indeed, contrary to most other clathrateforming molecules, the sublimation vapor pressure curve for pure $\mathrm{CO}_{2}$ ice (Giauque \& Egan 1937; Fray \& Schmitt 2009) lies just above the dissociation pressure curve of $\mathrm{CO}_{2}$ clathrate hydrate in equilibrium with $\mathrm{CO}_{2}$ gas $+\mathrm{H}_{2} \mathrm{O}$ ice. The different low-temperature extrapolations of these curves place the solid $\mathrm{CO}_{2} / \mathrm{H}_{2} \mathrm{O}$ ice $/ \mathrm{CO}_{2}$ clathrate $/ \mathrm{CO}_{2}$ gas quadruple point between 120 and $135 \mathrm{~K}$ (Miller \& Smythe 1970; Longhi 2005). The crossing of the curves is more probably around $131 \pm 2 \mathrm{~K}$. Our estimate is $130.2 \mathrm{~K}$ using Clausius-Clapeyron laws based on the first experimental determinations of these curves (Giauque \& Egan 1937; Miller \& Smythe 1970) as explained in the caption of Fig. 4. A more accurate determination based on compilations

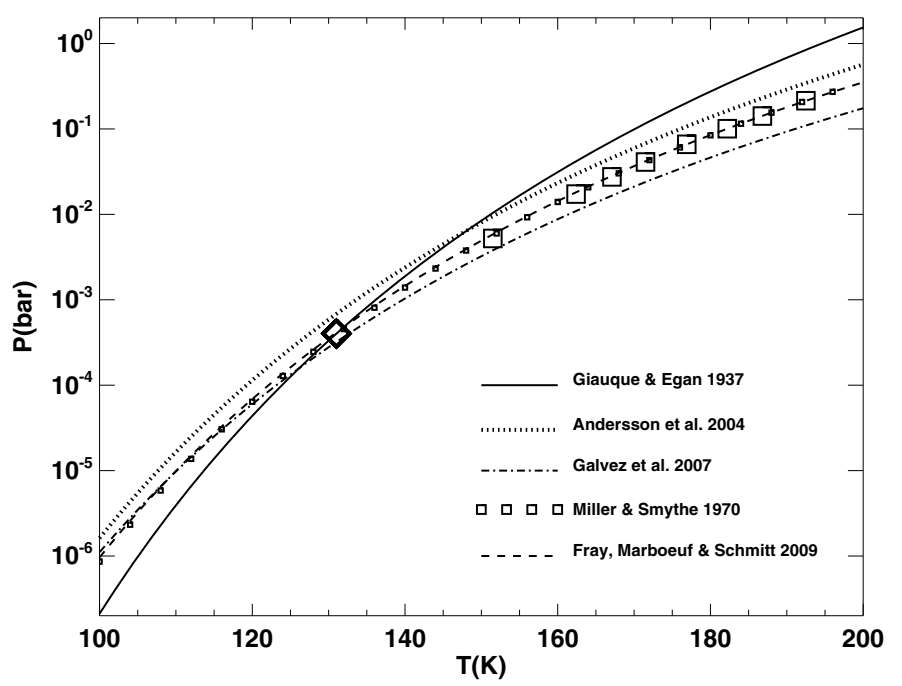

Fig. 4. Phase diagram for $\mathrm{CO}_{2}$ displaying the $\mathrm{CO}_{2}$ sublimation curve (black line, Giauque \& Egan 1937) and clathrate hydrate measurements (big squares, Miller \& Smythe 1970). Extrapolating the clathrate equilibrium experimental measurements with a Clausius-Clapeyron equation (small squares), we find the quadruple point at $130.2 \mathrm{~K}$. A recent compilation of $\mathrm{CO}_{2}$ clahrate hydrate dissociation pressures completed with measurements in the $170-190 \mathrm{~K}$ range by Fray et al. (2009) and extrapolated using a Clausius-Clapeyron equation is also displayed (dashed line) and leads to $132.5 \mathrm{~K}$ for the quadruple point. The mean value for the quadrupole point is indicated by a diamond. Two carbon dioxide adsorption equilibrium curves on crystalline ice surfaces, for $50 \%$ of the site coverage, based on measurements by Andersson et al. (2004) (thick dotted line) and Galvez et al. (2007) (dot-dashed line) are added to show the difficulty of phase determination from pressure measurements in this temperature range.

and critical reviews of the ice vapor pressures (Fray \& Schmitt 2009) and clathrate hydrate dissociation pressures (Fray et al. 2009) leads to a value of $132.5 \pm 1 \mathrm{~K}$.

\section{Discussion}

\section{1. $\mathrm{CO}_{2}$ clathrate in the solar system}

\section{Mars}

The proximity of the stability fields of the pure $\mathrm{CO}_{2}$ ice and its clathrate hydrate phases makes predicting their occurrence a difficult task in the context of the Martian surface where seasonal variations in surface temperature and atmospheric pressure can induce local and seasonal formation and decomposition of $\mathrm{CO}_{2}$ clathrate hydrate (e.g. Longhi 2006; Schmitt et al. 2003). To add to the complexity of the situation, the adsorption energy for $\mathrm{CO}_{2}$ on crystalline ice is rather high $\left(E_{\mathrm{A}} / k \sim 2553 \mathrm{~K}\right.$, Andersson et al. 2004; $E_{\mathrm{A}} / k \sim 2394 \mathrm{~K}$, Galvez et al. 2007). Then, if cold water ice particles are present in clouds or on the surface and their size distribution is dominated by very small particles displaying a high surface-to-volume ratio, $\mathrm{CO}_{2}$ adsorption may compete with the two previous phases mentioned. Considering these thermodynamical proximities, together with the kinetics behaviour of carbon dioxide interaction with ice (e.g. Schmitt 1986; Adamson \& Jones 1971), a spectroscopic discrimination is therefore an essential resource for distinguishing which phase actually occurs.

\section{Outer solar system}

Carbon dioxide is also present on many solar system icy bodies. The pure ice form is observed in the near-infrared from the 
Table 1. Carbon dioxide: gas, pure solid, hydrate, and clathrate hydrate transitions.

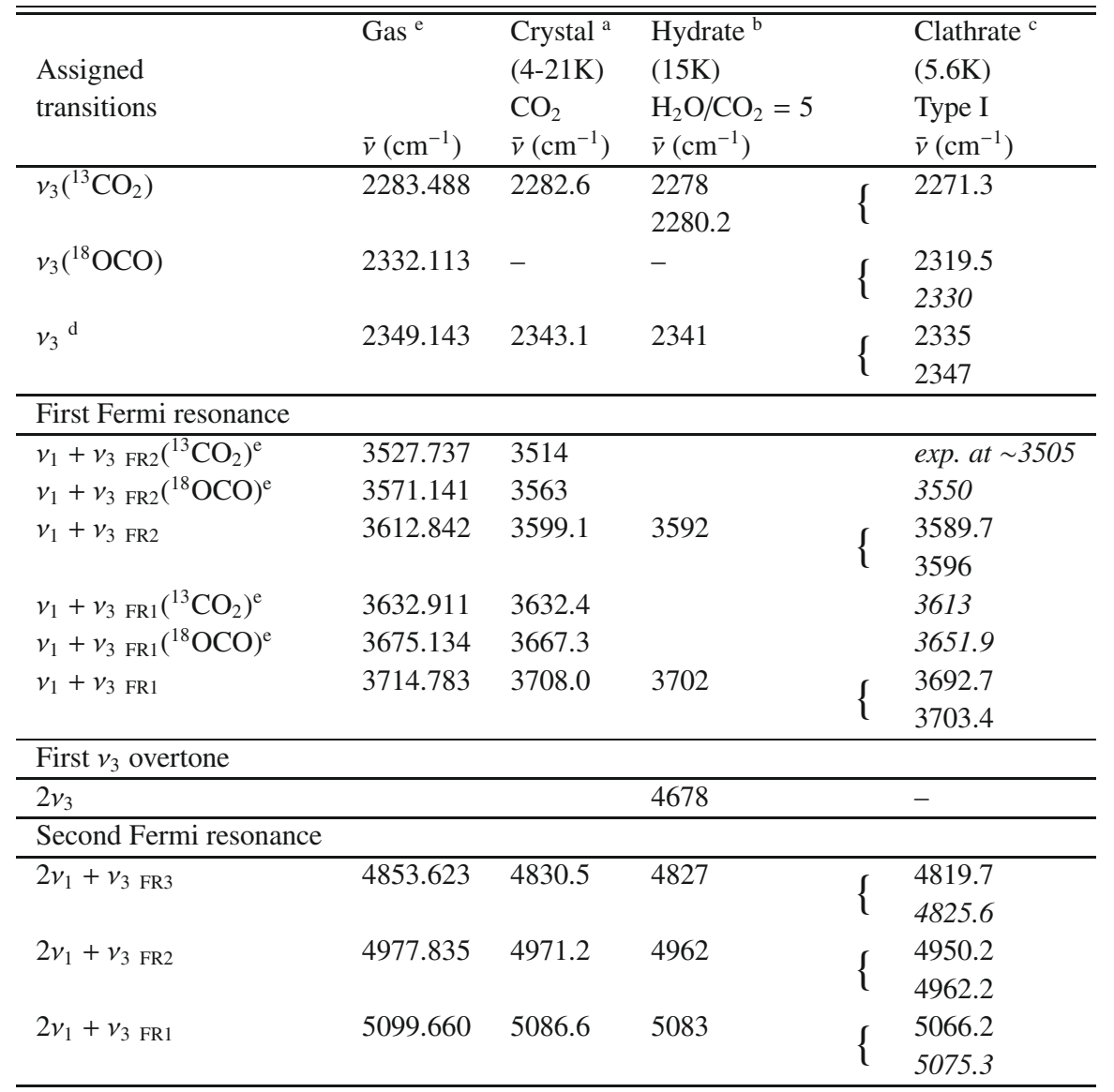

${ }^{a}$ Mid-infrared : pure $\mathrm{CO}_{2}$ spectrum measured at $4 \mathrm{~K}$ in April 1996 with the same spectrometer; Near-infrared spectra at $21 \mathrm{~K}$ and assignments from Quirico \& Schmitt (1997); ${ }^{\mathrm{b}}$ from Bernstein et al. (2005); ${ }^{\mathrm{c}}$ position accuracy is estimated to better than $0.5 \mathrm{~cm}^{-1}$, line positions in italics have higher uncertainties due to line blending, weakness, or saturation effects; ${ }^{\mathrm{d}}$ clathrate antisymmetric stretching-mode assignments for the $v_{3}\left({ }^{12} \mathrm{CO}_{2}\right)$ based on Fleyfel \& Devlin (1991); ${ }^{\mathrm{e}}$ transitions are assigned based on Rothman (1986).

second Fermi resonances on the surface of some satellites (on Ariel, Umbriel, Titania; Grundy et al. 2006, on Triton; Quirico et al. 1999) where the observed $\mathrm{H}_{2} \mathrm{O}$ ice is crystalline. Midinfrared reflectance spectra of the antisymmetric $\mathrm{CO}_{2}$ stretching mode display blue-shifted bands with respect to pure $\mathrm{CO}_{2}$ on Ganymede, Callisto (Hibbitts et al. 2000; McCord et al. 1998), Phoebe, Iapetus, and Hyperion (Buratti et al. 2005; Clark et al. 2005), which could be explained by the formation of hydrates (or complexes), e.g. Chaban et al. (2007) or by $\mathrm{CO}_{2}$ physisorbed on refractory solids (such as minerals, e.g. Hibbitts \& Szanyi 2007). On Triton the position and shape of the bands are consistent with pure $\mathrm{CO}_{2}$ ice, although very small shifts remain unexplained (Quirico et al. 1999).

\section{2. $\mathrm{CO}_{2}$ clathrate in the Interstellar Medium}

Although water ice largely dominates interstellar ice abundances, it is immediately followed by $\mathrm{CO}_{2}$ with a fairly constant $\mathrm{CO}_{2} / \mathrm{H}_{2} \mathrm{O}$ ratio around $20 \%$. In the interstellar medium, infrared observations have shown that $\mathrm{CO}_{2}$ is either segregated from the $\mathrm{H}_{2} \mathrm{O}$ ice mantles in the highest temperature, evolved protostellar envelopes (e.g. Keane et al. 2001), forming a molecular complex with methanol (e.g. Dartois et al. 1999; Ehrenfreund et al. 1999), or intermixed with $\mathrm{H}_{2} \mathrm{O}$ as a hydrate (e.g Gerakines et al. 1999; Pontoppidan et al. 2008, and references therein). However it may be possible that in some situations part of the $\mathrm{CO}_{2}$ may be trapped in a clathrate hydrate structure. Only spectroscopic evidence in the fundamental bands may allow identification of this phase. However, up to now the numerous infrared observations of $\mathrm{CO}_{2}$ in interstellar ice mantles do not display the characteristic double profile expected for clathrates.

\subsection{Detecting clathrates}

FTIR spectra of the type I carbon dioxide clathrate hydrate were recorded in the near to mid-infrared in the 5.6 to $150 \mathrm{~K}$ range. Characteristic double-peak profiles are observed, attributed to the same transition, but for $\mathrm{CO}_{2}$ trapped in the two cage types, small and large, of the structure I clathrate hydrate. Based on these spectroscopic profiles and on the positions of the two components, the occurrence of $\mathrm{CO}_{2}$ clathrate hydrate can be firmly constrained by remote spectroscopy of icy bodies either in the solar system or in the interstellar medium.

Reflectance (transmission) spectroscopy of icy surfaces of planets, satellites, or comets with spectral resolutions above about one thousand in the near(mid-)infrared would allow proper separation of the cages' distinct transitions. Such direct remote observations may result, however difficult to succeed. Clathrates may escape detection by being buried under the very upper surface layers, for stability reasons. A focus on thermodynamically 
favourable zones, or recently exposed (by e.g. an impact) surfaces, or else sublimating surfaces should be performed. This difficulty of observing clathrates has probably motivated thermodynamic approaches to determining their presence, as well as studies dedicated to their impact on the elemental or molecular abundance release. However, if such conditions for their formation and stability must be met, they should not be overused and stringent constraints fulfilled, such as their unambiguous spectroscopic detection.

In the light of expected cages occupancies, hence band doubling, no direct evidence of carbon dioxide clathrate hydrate has been reported so far in astrophysical objects, including our solar system. Therefore, as many different physical interactions are possible between $\mathrm{CO}_{2}$ (and many other molecules) and water ice, not necessarily involving the formation of a crystallographic system such as clathrate hydrates, it is important to be able to directly constrain the abundances and state of the various phases of $\mathrm{CO}_{2}$ in astrophysical media by spectroscopic means. This should help in understanding whether clathrate hydrates are widely present in astrophysical media or only locally in a few objects, whether its formation is ubiquitous given the widespread occurrence of carbon dioxide and water ice in astrophysical objects.

Acknowledgements. We wish to acknowledge in particular fruitful discussions and/or technical support from D. Barbet, M. Bouzit, A. Chardin, N. Coron, B. Crane, J. Crovisier, C. Dumesnil, J.-J. Fourmond, N. Fray, O. Brissaud, L. d'Hendecourt, T. Redon, G.Renoux and J. Adams.

\section{References}

Adamson, A. W., Jones B. R. 1971, J. Colloid Interface Sci., 37 (4), 831 Andersson, P. U., Ngrd, M. B., Witt G., \& Pettersson J. B. C. 2004, J. Phys. Chem. A, 108 (21), 4627

Barr, A. C., \& Milkovich, S. M. 2008, Icarus, 194, 513

Bernstein, M., Cruikshank, D. P., \& Sandford S. A. 2005, Icarus, 179, 527

Bockelée-Morvan, D., Crovisier, J., Mumma, M. J., \& Weaver, H. A. 2005a, in Comets II, ed. M. C. Festou, H. U. Keller, \& H. A. Weaver (Tucson: Univ. Arizona Press), 391

Boynton, W. V., Feldman, W. C., Squyres, S. W., et al. 2002, Science, 297, 81

Brown, R. H., Baines, K. H., Bellucci, G., et al. 2006, A\&A, 446, 707

Buratti, B. J., 28 colleagues, 2005. ApJ, 622, L149

Chaban, G. M., Bernstein, M., \& Cruikshank, D. P. 2007, Icarus, 187, 592

Circone, S., et al. 2003, J. Phys. Chem. B 107, 5529
Clark, R. N., Brown, R. H., Jaumann, R., et al. 2005, Nature, 435, 66 Colangeli, L., Epifani, E., Brucato, J. R., et al. 1999, A\&A, 343, L87

Combes, M., Crovisier, J., Encrenaz, T., Moroz, V. I., \& Bibring, J.-P. 1988, Icarus, 76, 404

Crovisier, J., Leech, K., Bockelee-Morvan, D., et al. 1997, Science, 275, 1904 Dartois, E., \& Deboffle D. 2008, 490, L19

Dartois, E., Demyk, K., d'Hendecourt, L., \& Ehrenfreund, P. 1999, A\&A, 351, 1066

de Graauw, T., Whittet, D. C. B., Gerakines, P. A., et al. 1996, A\&A, 315, L345 Demidov, N. E., Boynton, W. V., Gilichinsky, D. A., et al. 2008, Astron. Lett., 34,713

Ehrenfreund, P., Kerkhof, O., Schutte, W. A., et al. 1999, A\&A, 350, 240

Feaga, L. M., A’Hearn, M. F., Sunshine, J. M., Groussin, O., \& Farnham, T. L. 2007, Icarus, 190, 345

Feldman, P. D., Festou, M. C., Tozzi, G. P., \& Weaver, H. A. 1997, ApJ, 475, 829

Fleyfel, F., \& Devlin, J.-P. 1991, J. Phys Chem., 95, 5, 3811

Fray N., \& Schmitt B. 2009, Planet. \& Space Sci., submitted

Fray, N., Marboeuf, U., \& Schmitt, B., Planet. Space Sci., accepted

Gálvez, O., Ortega, I. K., Maté, B., et al. 2007, A\&A, 472, 691

Gerakines, P. A., Whittet, D. C. B., Ehrenfreund, P., et al. 1999, ApJ, 522, 357

Genov, G., \& Kuhs, W. F. 2003, Third International Conference on Mars Polar Science and Exploration, 8011

Giauque, W. F., \& Egan, C. J. 1937, J. Chem. Phys., 5, 45

Grundy, W. M., Young, L. A., Spencer, J. R. 2006, Icarus, 184, 543

Hibbitts, C. A., \& Szanyi, J. 2007, Icarus, 191, 371

Hibbitts, C. A., McCord, T. B., \& Hansen, G. B. 2000, J. Geophys. Res. 105, 2254

Hibbitts, C. A., Pappalardo, R. T., Hansen, G. B., \& McCord, T. B. 2003, J. Geophys. Res. (Planets), 108, 5036

Keane, J. V., Boogert, A. C. A., Tielens, A. G. G. M., Ehrenfreund, P., \& Schutte, W. A. 2001, A\&A, 375, L43

Longhi, J. 2005, Geochim. Cosmochim. Acta, 69 (3), 529

Longhi, J. 2006, J. Geophys. Res. (Planets), 111, 6011

Lunine, J. I., \& Stevenson D. J. 1985, ApJS, 58, 493

McCord, T. B., et al. 1998, J. Geophys. Res., 103, 8603

Miller, S. L., \& Smythe, W. D. 1970, Science, 170, 531

Mouginot, J., Beck, P., Pommerol, A., et al. 2009, Nature, submitted

Pontoppidan, K. M., et al. 2008, ApJ, 678, 1005

Quirico E., \& Schmitt B. 1997, Icarus, 127, 354

Quirico, E., Douté, S., Schmitt, B., et al. 1999, Icarus, 139, 159

Rothman, L. S. 1981, Appl. Opt., 25, 1795

Rothman, L. S., \& Young, L. D. G. 1981, J. Quant. Spectrosc. Radiat. Transf., 25,505

Schmitt, B., \& Thesis 1986, The surface of ice: structure, dynamics and interactions. Astrophysical implications, Université de Grenoble

Schmitt, B., Mulato, L., \& Douté, S. 2003, Third International Conference on Mars Polar Science and Exploration, 8073

Tamppari, L. K., Smith, M. D., Bass, D. S., \& Hale, A. S. 2008, Planet. Space Sci., 56, 227

Weaver, H. A., Feldman, P. D., McPhate, J. B., et al. 1994, ApJ, 422, 374 